\title{
Water masers in the massive protostar IRAS 20126+4104: ejection and deceleration
}

\author{
L. Moscadelli ${ }^{1}$, R. Cesaroni ${ }^{2}$, and M. J. Rioja ${ }^{3}$ \\ 1 INAF, Osservatorio Astronomico di Cagliari, Loc. Poggio dei Pini, strada 54, 09012 Capoterra (Cagliari), Italy \\ e-mail: mosca@ca.astro.it \\ 2 INAF, Osservatorio Astrofisico di Arcetri, Largo E. Fermi 5, 50125 Firenze, Italy \\ 3 Observatorio Astronomico Nacional (IGN), Apartado 1143, 28800 Alcalá de Henares (Madrid), Spain
}

Received 12 January 2005 / Accepted 29 March 2005

\begin{abstract}
We report on the first multi-epoch, phase referenced VLBI observations of the $\mathrm{H}_{2} \mathrm{O}$ maser emission in a highmass protostar associated with a disk-jet system. The source under study, IRAS 20126+4104, has been extensively investigated in a large variety of tracers, including $\mathrm{H}_{2} \mathrm{O}$ maser VLBA data acquired by us three years before the present observations. The new findings fully confirm the interpretation proposed in our previous study, namely that the maser spots are expanding from a common origin coincident with the protostar. We also demonstrate that the observed 3-D velocities of the maser spots can be fitted with a model assuming that the spots are moving along the surface of a conical jet, with speed increasing for increasing distance from the cone vertex. We also present the results of single-dish monitoring of the $\mathrm{H}_{2} \mathrm{O}$ maser spectra in IRAS 20126+4104. These reveal that the peak velocity of some maser lines decreases linearly with time. We speculate that such a deceleration could be due to braking of the shocks from which the maser emission originates, due to mass loading at the shock front or dissipation of the shock energy.
\end{abstract}

Key words. masers - ISM: jets and outflows - ISM: individual objects: IRAS 20126+4104

\section{Introduction}

Several molecular species are known to exhibit maser emission from the sites of newly born stars (see e.g. Elitzur 1992). This applies especially to the regions where high-mass stars (i.e. stars in excess of $\sim 8 M_{\odot}$ ) form. In particular, the water line at $22.2 \mathrm{GHz}$, originating from the $6_{16} \rightarrow 5_{23}$ transition, is the most powerful emitter, attaining flux densities up to $10^{6} \mathrm{Jy}$ in a $\sim 1 \mathrm{~km} \mathrm{~s}^{-1}$ wide line. Since the emitting region (named "spot") of a maser line is as small as 1 mas or less, $\mathrm{H}_{2} \mathrm{O}$ masers are excellent tools for high angular resolution and low sensitivity observations such as those performed with very long baseline interferometry (VLBI). These characteristics make it possible to use $\mathrm{H}_{2} \mathrm{O}$ masers as "test particles" to trace the velocity field of the gas in the densest and hence most obscured portions of molecular clouds. This is of great help in studies of massive star forming regions, which are hindered by two problems: massive stars are much more distant (a few kpc) than low-mass stars and are born deeply embedded in their parental cores. Both shortcomings are overcome by $\mathrm{H}_{2} \mathrm{O}$ maser observations: on the one hand, VLBI observations can attain angular resolutions of $\sim 1$ mas, corresponding to $\sim 1$ au at $1 \mathrm{kpc}$; on the other hand, observations at $22 \mathrm{GHz}$ may easily penetrate even the highest column densities.

For these reasons, $\mathrm{H}_{2} \mathrm{O}$ masers have been extensively used to pin-point the location of young stellar objects (YSOs) and investigate the structure and kinematics of the gas in their surroundings. Although some authors have suggested that such masers might originate in circumstellar disks (see e.g. Torrelles et al. 1996, 1998; Goddi et al. 2004), the common belief is that they are associated with outflows (Felli et al. 1992). In one case (Torrelles et al. 2001), the maser spots have been found to describe a perfect circle (to one part in a thousand), suggesting that they could originate at the interface between a spherical stellar wind from a deeply embedded YSO and the surrounding gas.

With this in mind, Moscadelli et al. (2000; hereafter MCR) performed 1 mas resolution observations with the very large baseline array (VLBA) of the $\mathrm{H}_{2} \mathrm{O}$ maser emission in the wellknown massive YSO IRAS 20126+4104, which is the best example to date of a high-mass protostar associated with a Keplerian disk and bipolar outflow/jet (Cesaroni et al. 1997, 1999, 2005; Zhang et al. 1998; Hofner et al. 1999; Shepherd et al. 2000). The scope of the observations was to establish if the masers were co-rotating with the Keplerian disk or expanding with the outflow/jet. The former possibility was ruled out by MCR, who demonstrated that the $\mathrm{H}_{2} \mathrm{O}$ maser spots are distributed along the axis of the outflow/jet and may be adequately fitted with a conical model implying an expansion velocity of $\sim 23 \mathrm{~km} \mathrm{~s}^{-1}$. Such findings are of great importance for a better understanding of the structure of the jet in IRAS 20126+4104, which is known to be undergoing 
precession (Shepherd et al. 2000). Consequently, to establish the current direction of the jet it is necessary to observe it as close as possible to the powering source (see Cesaroni et al. 2005 , for a thorough discussion of this topic), which may be best achieved with a VLBI study of $\mathrm{H}_{2} \mathrm{O}$ masers.

Notwithstanding the results obtained by MCR, their study was limited to the l.o.s. component of the velocity of the spots. This is a serious limitation in the case of IRAS 20126+4104 because the jet axis lies very close to the plane of the sky (Cesaroni et al. 1999), thus making an accurate estimate of the expansion velocity very difficult. A small error on the estimate of the inclination of the velocity vector may be reflected as a large uncertainty on the true expansion speed. In order to overcome this problem and hence obtain a more accurate description of the jet, we have performed new multiepoch observations with global VLBI, which resulted in proper motion measurements of the $\mathrm{H}_{2} \mathrm{O}$ maser spots. In the following we present the results of this study and improve the model fit proposed by MCR to take into account the spots' motion in the plane of the sky. Finally, we present the results of single-dish monitoring of the $\mathrm{H}_{2} \mathrm{O}$ maser emission from IRAS $20126+4104$ over a period of $\sim 15$ years and interpret these in the light of the VLBI findings.

\section{Observations and data reduction}

IRAS 20126+4104 was observed using the Global array (including a subset of $\mathrm{EVN}^{1}$ antennae plus the 10 antennae of the $\mathrm{VLBA}^{2}$ ) for $18 \mathrm{~h}$ at three epochs, on November 9 and 26, 2000, and on March 1, 2001. A group of 6 EVN antennae (Effelsberg, Jodrell, Medicina, Noto, Onsala, and Shanghai) took part in the observations at each epoch, whereas Metsähovi and Robledo observed only the first two epochs and the second epoch, respectively. The observations were performed in phasereferencing mode alternating scans on the maser target with scans of the phase-reference source J2007+4029, with a cycle time of $\approx 1 \mathrm{~min}$. $\mathrm{J} 2007+4029$ is an intense quasar belonging to the ICRF (International Celestial Reference Frame) list, with a relative separation from the target maser source of 1.5. For the purpose of bandpass and phase calibration, a scan of a few minutes on one of the strong, compact calibrators J2007+777 and $\mathrm{J} 2002+4725$, was observed every $1-2 \mathrm{~h}$.

The stations recorded an aggregate of $16 \mathrm{MHz}$ bandwidth in both circular polarizations, centered at the LSR velocity of $-3.5 \mathrm{~km} \mathrm{~s}^{-1}$ (based upon a rest frequency of $22235.0798 \mathrm{MHz}$ ), using 2-bit sampling. The correlation was made at the VLBA correlator in Socorro (New Mexico) using 1024 spectral channels uniformly weighted, which gives a channel separation of $0.21 \mathrm{~km} \mathrm{~s}^{-1}$.

The amplitude and phase calibration of the observed visibilities and the mapping were accomplished with the NRAO AIPS package, following the same procedure described

\footnotetext{
1 The European VLBI Network is a joint facility of European, Chinese, South African and other radio astronomy institutes funded by their national research councils.

2 The VLBA is a facility of the NRAO, which is operated by Associated Universities, Inc., under contract with the NSF.
}

by MCR. At each epoch, the maser phase-reference channel was chosen to be the one with the strongest emission, whose velocity changes slightly across the epochs (from $-14.7 \mathrm{~km} \mathrm{~s}^{-1}$ to $-15.5 \mathrm{~km} \mathrm{~s}^{-1}$ ), and whose structure consists of a single, unresolved spot. The absolute position of the reference maser spot has been derived from the maps produced after applying the corrections calculated using the phase-reference source data. These corrections were obtained in two steps. First, after applying the calibrator corrections, the visibilities of the phase-reference source were fringe fitted to find the residual fringe rate produced both by differences in atmospheric fluctuations between the calibrators and the source, and by errors in the model used at the correlator. Then, after correcting for the residual fringe rate, the visibilities of the phase-reference source were self-calibrated to remove any possible effect induced by extended spatial structure. For the first observing epoch, the absolute position of the reference maser spot is: $\mathrm{RA}(\mathrm{J} 2000)=20^{\mathrm{h}} 14^{\mathrm{m}} 26^{\mathrm{s}} 0253, \operatorname{Dec}(\mathrm{J} 2000)=41^{\circ} 13^{\prime} 32^{\prime \prime} .666$, estimated to be accurate within a few tenths of milliarcsecond. As a check, we have also calculated the absolute position with the reverse phase-referencing technique (i.e. mapping the phase-reference source after applying the corrections evaluated working with the maser data), and found fully consistent results.

Using the AIPS task "IMAGR", 1 " $\times 1$ " $(\mathrm{E} \times \mathrm{N})$ tapered, channel-averaged maps centered on the reference spot were produced for the velocity range from -28 to $+3 \mathrm{~km} \mathrm{~s}^{-1}$, which includes all the emission apparent in the total power spectra. We note that, at each epoch, the maser emission extends over a velocity range narrower than that of the VLBA observations by MCR, for which spectral features in the velocity interval from +3 to $+15 \mathrm{~km} \mathrm{~s}^{-1}$ were also observed. The field of view covered by the tapered maps is about twice the sky area over which water maser emission is detected in the MCR's maps. The bulk of maser emission is found within a distance of $\approx 200$ mas from the reference spot, with the exception of a single feature detected at a distance of $~ 530$ mas. In order to map the maser emission at full angular and velocity resolution, at each epoch and for each velocity channel in the range from -32 to $+10 \mathrm{~km} \mathrm{~s}^{-1}$, we produced 0.' $4 \times 00^{\prime} .4(\mathrm{E} \times \mathrm{N})$ naturally weighted maps centered on the reference spot. In addition to that, smaller maps were also created at the position and velocity of the more detached feature. The CLEAN beam was an elliptical Gaussian with a full width at half maximum $(F W H M)$ size, slightly varying from epoch to epoch, of 0.6-0.8 mas along the major axis and 0.5-0.6 mas along the minor axis. In each observing epoch, the $1 \sigma$ rms noise level on the channel maps is close to the theoretical thermal value, $3 \mathrm{mJy}$ beam $^{-1}$, for channels where no signal is detected, and increases to $13 \mathrm{mJy}^{\text {beam }}{ }^{-1}$ for channels with the strongest components.

Every channel map was searched for emission above a conservative detection threshold (in the range 5-15 $\sigma$ ), and the detected maser spots were fitted with two-dimensional elliptical Gaussians, determining position, flux density, and FWHM size of the emission. Hereafter, we use the term "spot" to indicate the mean position of a collection of spectrally and spatially contiguous maser spots. As the typical line width of the water 
Table 1. Parameters of the water maser spots in IRAS $20126+4104$.

\begin{tabular}{|c|c|c|c|c|c|c|c|c|}
\hline Number & $\begin{array}{c}\Delta \mathrm{RA}^{(a)} \\
\text { (mas) }\end{array}$ & $\begin{array}{c}\Delta \operatorname{Dec}^{(a)} \\
(\mathrm{mas})\end{array}$ & $\begin{array}{l}S_{v} \\
(\mathrm{Jy})\end{array}$ & $\begin{array}{c}V_{\mathrm{LSR}} \\
\left(\mathrm{km} \mathrm{s}^{-1}\right)\end{array}$ & $\begin{array}{c}V_{\mathrm{RA}}^{(b)} \\
\left(\mathrm{km} \mathrm{s}^{-1}\right)\end{array}$ & $\begin{array}{c}V_{\operatorname{Dec}^{(b)}} \\
\left(\mathrm{km} \mathrm{s}^{-1}\right)\end{array}$ & $\begin{array}{c}V_{\mathrm{RA}}^{(c)} \\
\left(\mathrm{km} \mathrm{s}^{-1}\right)\end{array}$ & $\begin{array}{c}V_{\text {Dec }}{ }^{(c)} \\
\left(\mathrm{km} \mathrm{s}^{-1}\right)\end{array}$ \\
\hline 1 & $0.0 \pm 0.3$ & $0.0 \pm 0.3$ & 24.30 & -15.0 & $-75 \pm 10$ & $17 \pm 10$ & $-84 \pm 10$ & $54 \pm 10$ \\
\hline 2 & $-1.0 \pm 0.3$ & $0.5 \pm 0.3$ & 18.36 & -15.3 & - & - & - & - \\
\hline 3 & $-2.3 \pm 0.3$ & $1.2 \pm 0.3$ & 10.94 & -16.2 & - & - & - & - \\
\hline 4 & $-1.6 \pm 0.3$ & $0.8 \pm 0.3$ & 5.12 & -16.0 & - & - & - & - \\
\hline 5 & $-24.5 \pm 0.3$ & $-51.5 \pm 0.3$ & 4.50 & -2.3 & $-39 \pm 11$ & $-12 \pm 10$ & $-48 \pm 11$ & $25 \pm 10$ \\
\hline 6 & $-21.5 \pm 0.3$ & $-65.8 \pm 0.3$ & 5.58 & -3.3 & $-57 \pm 11$ & $-23 \pm 11$ & $-66 \pm 11$ & $14 \pm 11$ \\
\hline 7 & $-21.1 \pm 0.3$ & $-66.6 \pm 0.3$ & 0.92 & -5.1 & $-48 \pm 12$ & $-23 \pm 11$ & $-58 \pm 12$ & $14 \pm 11$ \\
\hline 8 & $-22.9 \pm 0.3$ & $-63.0 \pm 0.3$ & 0.47 & -5.8 & $-42 \pm 11$ & $-19 \pm 10$ & $-52 \pm 11$ & $19 \pm 10$ \\
\hline 9 & $-28.0 \pm 0.3$ & $-60.4 \pm 0.3$ & 5.77 & -8.5 & $-46 \pm 11$ & $-20 \pm 11$ & $-55 \pm 11$ & $17 \pm 11$ \\
\hline 10 & $-25.8 \pm 0.3$ & $-61.6 \pm 0.4$ & 2.97 & -9.5 & $-43 \pm 12$ & $-33 \pm 11$ & $-53 \pm 12$ & $4 \pm 11$ \\
\hline 11 & $-26.0 \pm 0.3$ & $-60.9 \pm 0.3$ & 2.08 & -8.6 & $-43 \pm 11$ & $-21 \pm 11$ & $-52 \pm 11$ & $16 \pm 11$ \\
\hline 12 & $-9.2 \pm 0.3$ & $0.1 \pm 0.3$ & 0.29 & -14.8 & $-67 \pm 11$ & $19 \pm 10$ & $-76 \pm 11$ & $56 \pm 10$ \\
\hline 13 & $12.3 \pm 0.3$ & $-83.8 \pm 0.3$ & 0.24 & -5.6 & $-33 \pm 11$ & $-31 \pm 11$ & $-42 \pm 11$ & $7 \pm 11$ \\
\hline 14 & $11.7 \pm 0.3$ & $-84.9 \pm 0.3$ & 0.08 & -3.7 & $-35 \pm 12$ & $-20 \pm 11$ & $-44 \pm 12$ & $17 \pm 11$ \\
\hline 15 & $14.8 \pm 0.4$ & $24.4 \pm 0.4$ & 0.49 & -8.0 & $-19 \pm 12$ & $-19 \pm 11$ & $-28 \pm 12$ & $18 \pm 11$ \\
\hline 16 & $-141.9 \pm 0.3$ & $73.0 \pm 0.3$ & 0.85 & -24.9 & $-76 \pm 11$ & $9 \pm 10$ & $-85 \pm 11$ & $46 \pm 10$ \\
\hline 17 & $-142.6 \pm 0.3$ & $73.2 \pm 0.3$ & 0.09 & -24.8 & - & - & - & - \\
\hline 18 & $-17.8 \pm 0.3$ & $-67.0 \pm 0.3$ & 0.17 & -9.5 & $-61 \pm 11$ & $-25 \pm 10$ & $-70 \pm 11$ & $12 \pm 10$ \\
\hline 19 & $-19.0 \pm 0.3$ & $-66.4 \pm 0.3$ & 0.14 & -7.9 & $-49 \pm 11$ & $-28 \pm 10$ & $-59 \pm 11$ & $9 \pm 10$ \\
\hline 20 & $2.1 \pm 0.3$ & $-0.7 \pm 0.3$ & 0.13 & -14.2 & $-66 \pm 11$ & $46 \pm 10$ & $-76 \pm 11$ & $83 \pm 10$ \\
\hline 21 & $6.4 \pm 0.3$ & $29.6 \pm 0.3$ & 0.12 & -8.0 & - & - & - & - \\
\hline 22 & $29.9 \pm 0.3$ & $-77.1 \pm 0.3$ & 0.16 & -3.8 & - & - & - & - \\
\hline 23 & $28.9 \pm 0.3$ & $-78.2 \pm 0.3$ & 0.13 & -3.8 & - & - & - & - \\
\hline 24 & $21.3 \pm 0.3$ & $-87.2 \pm 0.3$ & 0.06 & 2.7 & - & - & - & - \\
\hline 25 & $28.2 \pm 0.3$ & $-78.0 \pm 0.3$ & 0.10 & -3.8 & - & - & - & - \\
\hline 26 & $482.7 \pm 0.3$ & $-228.3 \pm 0.3$ & 1.06 & -1.9 & $7 \pm 11$ & $-48 \pm 10$ & $-2 \pm 11$ & $-11 \pm 10$ \\
\hline
\end{tabular}

(a) Offset with respect to $\mathrm{RA}(\mathrm{J} 2000)=20^{\mathrm{h}} 14^{\mathrm{m}} 26^{\mathrm{s}} .0253$, Dec $(\mathrm{J} 2000)=41^{\circ} 13^{\prime} 32^{\prime \prime} 666$.

(b) Absolute proper motion measured with phase referencing.

(c) Absolute intrinsic proper motion after correction for parallax, motion of the Sun with respect to the LSR, and galactic rotation.

masers is greater than $0.5 \mathrm{~km} \mathrm{~s}^{-1}$, a maser feature is considered real if it is detected in at least three contiguous channels $\left(0.21 \mathrm{~km} \mathrm{~s}^{-1}\right.$ wide $)$ at the same position, within an uncertainty equal to the $F W H M$ obtained with the Gaussian fit.

The uncertainty in the relative positions of the maser spots is estimated using the expression (Reid et al. 1988)

$\Delta \theta=\frac{\sigma}{2 I} F W H M$

where $F W H M$ is the un-deconvolved spot diameter, $I$ is the peak intensity and $\sigma$ is the rms of the map evaluated over a region where no signal is present. Depending on the spot intensity, the relative positional uncertainty varies in the range $\approx 0.1-100 \mu$ as.

\section{Results}

\subsection{Maser positions and velocities}

Table 1 lists the parameters of the detected maser spots. Column 1 gives the spot label number. Columns 2 and 3 report the positional (RA and Dec) offsets (measured on the first epoch of detection) calculated with respect to the reference spot (labeled \# 1). Such offsets are estimated from the (errorweighted) mean positions of the contributing maser spots. The positional uncertainties are evaluated by taking the weighted standard deviation of the spot positions. Columns 4 and 5 list respectively the integrated flux density, $S_{v}$, and the line-of-sight velocity, $V_{\mathrm{LSR}}$, of the spot highest-intensity channel, both averaged over the observational epochs for the time-persistent spots. 
The absolute position of a spot at each observing epoch is calculated adding the (RA and Dec) offsets to the absolute position of the reference spot. The absolute proper motions have been calculated performing a (error-weighted) linear least-squares fit of the absolute positions with time. Columns 6 and 7 of Table 1 report the projected components, respectively along the RA and Dec axis, of the measured absolute proper motions, together with the formal errors of the linear leastsquares fit. Using a distance to IRAS 20126+4104 of $1.7 \mathrm{kpc}$, Columns 8 and 9 report the absolute proper motion components after correction for parallax, motion of the Sun with respect to the LSR, and galactic rotation.

\subsection{The distance to IRAS $20126+4104$}

The absolute proper motions measured by us are the sum of the intrinsic proper motion due to the velocity of the maser spots with respect to the YSO and the apparent motion due to the annual parallax and motion of the source with respect to the Sun. Therefore, one of the possible outcomes of a proper motion study is the derivation of the distance to the source by fitting the annual parallax. However this technique cannot be applied in our case, as the few measurements (3) and the short intervals between them ( $\leq 95$ days) are insufficient to properly sample the apparent position of the spots as a function of time - at least 5 points evenly distributed over 1 year would be needed (see e.g. Vlemmings et al. 2003). Also, for the $\mathrm{H}_{2} \mathrm{O}$ masers in IRAS 20126+4104 the situation is complicated by the fact

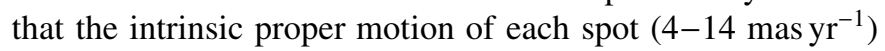
is greater (see Sect. 3.3) than that due to the annual paral$\operatorname{lax}\left(\lesssim 1.2\right.$ mas yr $\left.^{-1}\right)$.

The same considerations hold when deriving the intrinsic proper motion of each spot: the apparent proper motion due to the parallax and source velocity must be subtracted ${ }^{3}$ from the observed proper motion. Such an apparent motion depends on the distance and consequently also the estimate of the intrinsic motion depends on it. One may thus use this dependency to constrain the distance. Two approaches are possible.

The first uses the fact that, to a first order approximation, the maser spots should move along a given direction, since they are participating in the expansion of the jet from IRAS 20126+4104. This implies that for each spot the positions on the plane of the sky at the three epochs should lie along a straight line. A quantitative estimate of this is obtained from the correlation coefficient, $r$, of each triplet of positions per spot. We have hence computed $r$ for all spots and calculated the arithmetic mean. This is shown in Fig. 1 as a function of the distance, $d$. A maximum is reached for $d=1.4 \mathrm{kpc}$, but also larger distances cannot be excluded, whereas for $d<1 \mathrm{kpc}$ the correlation coefficient drops significantly.

An alternative approach is that of comparing the mean direction of the proper motions (after subtraction of the

\footnotetext{
3 The contribution of the parallax and source motion relative to the Sun have been calculated with a program kindly provided by Prof. Tetsuo Sasao. Both a flat galactic rotation curve and the rotation curve of Brand \& Blitz (1993) have been considered, but the results obtained for a distance of $1.7 \mathrm{kpc}$ were not significantly different.
}

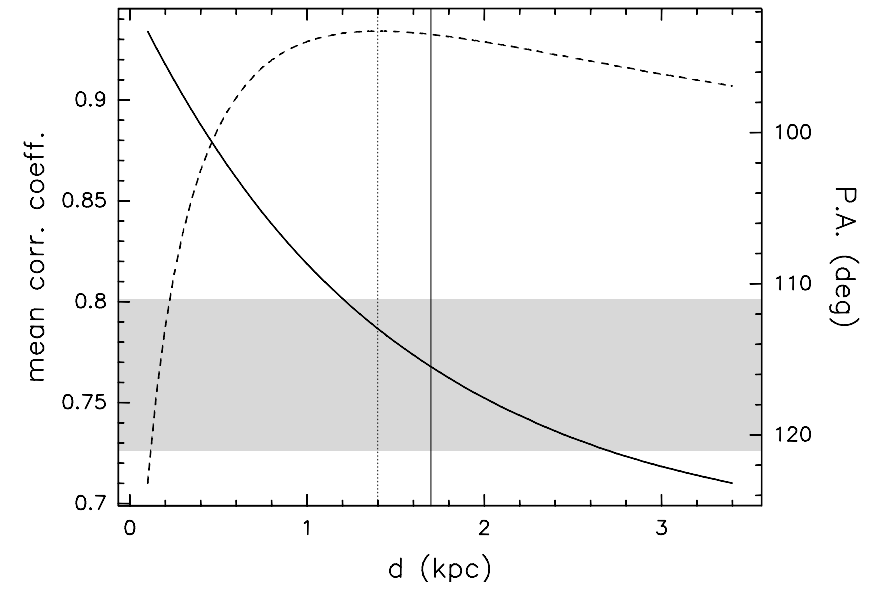

Fig. 1. Plot of mean correlation coefficient of the maser spots (dashed curve; see text) and mean direction of the normalised proper motions (solid curve) versus source distance. The vertical dotted and solid lines mark respectively $d=1.4$ and $1.7 \mathrm{kpc}$. The grey area denotes the range of possible jet position angles.

apparent motion) with the direction of the jet axis. To give equal weights to all spots we have performed a vector average of the unity vectors of the proper motions in Cols. 6 and 7 of Table 1, weighted by the corresponding errors. The result is shown in Fig. 1 as a function of the distance. The grey area in the same figure marks the range of plausible values of the jet position angle (PA), obtained from tracers such as the $\mathrm{SiO}(2-1)$ and $\mathrm{H}_{2}$ lines (Cesaroni et al. 1999), and the $2.2 \mu \mathrm{m}$ (Edris et al. 2005, hereafter EFCE) and $3.6 \mathrm{~cm}$ continuum emission (Hofner et al. 1999; Hofner, pers. comm.). Clearly, any distance between 1.2 and $2.7 \mathrm{kpc}$ is possible.

We conclude that with the present data only loose constraints can be set on the distance to IRAS 20126+4104, indicating that the source cannot be closer than $1.2 \mathrm{kpc}$. In the following we will assume the usual estimate of $1.7 \mathrm{kpc}$ for consistency with previous studies of the source.

\subsection{Distribution and velocities of the $\mathrm{H}_{2} \mathrm{O}$ maser spots}

As previously mentioned, the main scope of our study was to investigate the geometry and kinematics of the jet in IRAS $20126+4104$ using the $\mathrm{H}_{2} \mathrm{O}$ maser spots as test particles of the outflowing molecular gas. Figure $2 \mathrm{a}$ shows all the $\mathrm{H}_{2} \mathrm{O}$ maser spots detected in the region by us, MCR, and EFCE. Also shown are the $\mathrm{OH}$ maser emission peaks, which according to EFCE mark the plane of the circumstellar disk. We overlay the masers on a contour map of the $3.6 \mathrm{~cm}$ continuum emission imaged by Hofner et al. (1999), corresponding to the inner ionised part of the jet/outflow in IRAS 20126+4104. All of these tracers agree very well with the pattern of the conical jet model that will be discussed in Sect. 4 and is already presented here for the sake of comparison. As expected, the vertex of the cone - i.e. the putative position of the YSO powering the jet falls very close to the peak of the $3.6 \mathrm{~cm}$ continuum emission and in between the two $\mathrm{OH}$ maser peaks denoting the disk.

All this strongly favours the scenario already outlined by $\mathrm{MCR}$, according to which the $\mathrm{H}_{2} \mathrm{O}$ masers are located at 

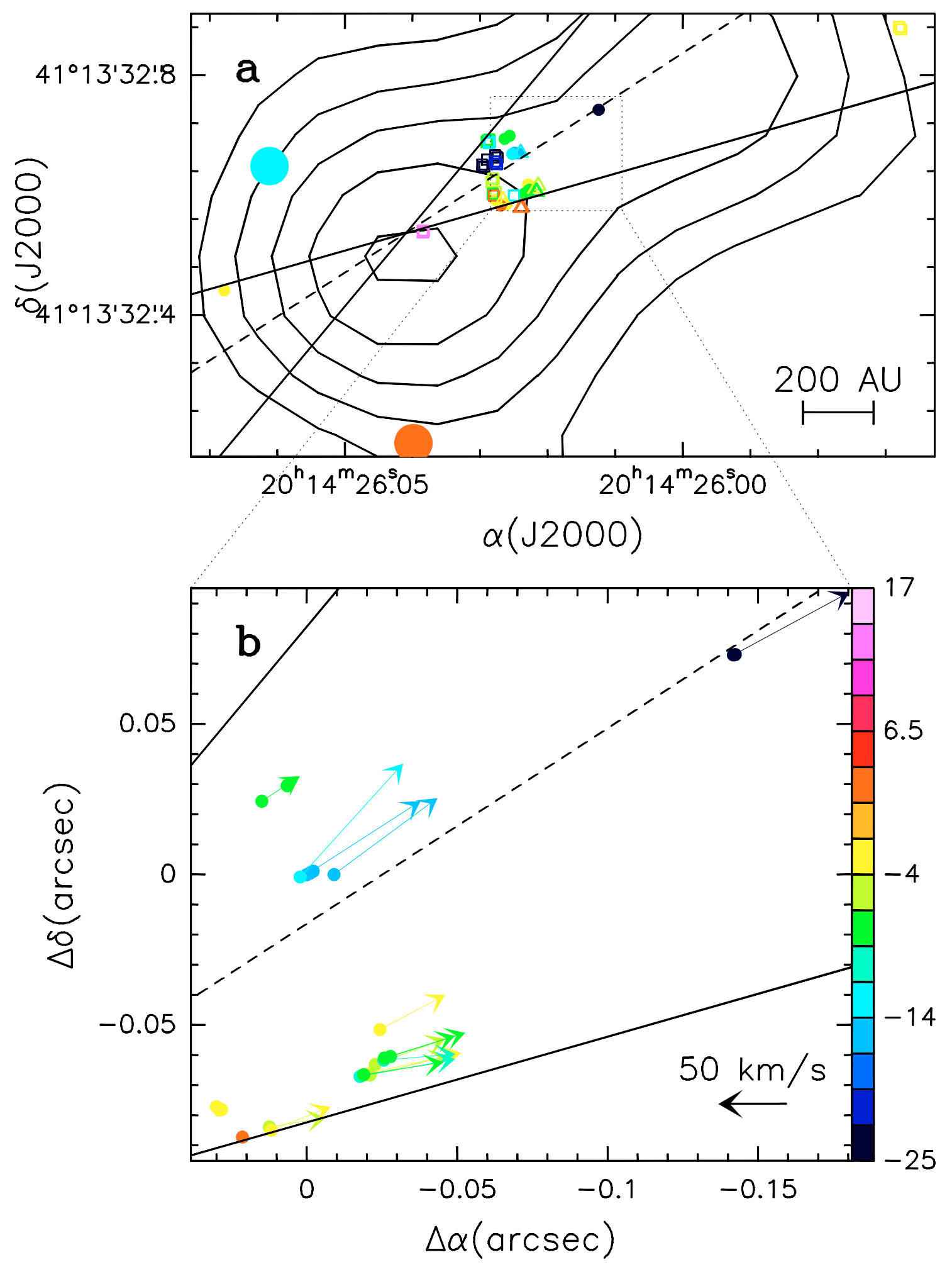

Fig. 2. a) Map of the $\mathrm{H}_{2} \mathrm{O}$ maser spots detected towards IRAS 20126+4104 by us (small circles), MCR (squares), and EFCE (triangles) overlayed on a contour map of the $3.6 \mathrm{~cm}$ continuum emission (Hofner et al. 1999). Also shown are the OH maser emission peaks observed by EFCE (big circles). The errors on the absolute positions of the $\mathrm{H}_{2} \mathrm{O}$ maser spots detected by us are given in Table 1, while those of MCR and EFCE are respectively 30 mas and 15 mas; for the $\mathrm{OH}$ masers the error is 25 mas. The colour denotes the LSR velocity of each spot according to the colour scale in the bottom panel. The solid lines indicate the conical jet which represents the best fit to the $\mathrm{H}_{2} \mathrm{O}$ maser spots obtained with the model discussed in Sect. 4. b) Enlargement of the central region illustrating the locations and absolute proper motions (corrected for parallax, solar motion with respect to the LSR, and galactic rotation) of the $\mathrm{H}_{2} \mathrm{O}$ maser spots detected in this study. Offsets in RA and Dec are measured with respect to $\mathrm{RA}(\mathrm{J} 2000)=20^{\mathrm{h}} 14^{\mathrm{m}} 26^{\mathrm{s}} .0253$, Dec(J2000) $=41^{\circ} 13^{\prime} 32^{\prime \prime}$. 666 . No proper motion measurement is available for points without an associated arrow. The colour coding is the same as for the top panel. Note that the systemic LSR velocity of IRAS $20126+4104$ is $-3.5 \mathrm{~km} \mathrm{~s}^{-1}$. 


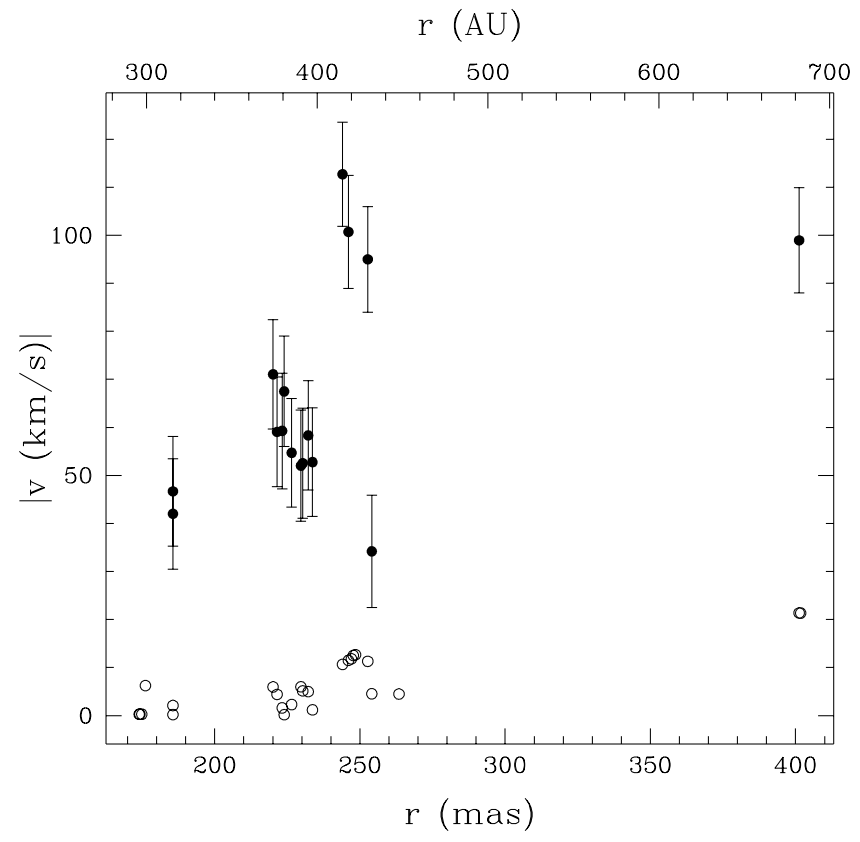

Fig. 3. Plot of speed versus separation (measured on the plane of the sky) from the $3.6 \mathrm{~cm}$ continuum peak for all $\mathrm{H}_{2} \mathrm{O}$ maser spots of Fig. 2b with measured proper motions (filled circles). Also shown is a plot of the l.o.s. component of the velocity for all spots detected in this study (empty circles). Note the tendency of the speed to increase with distance from the YSO.

the interface between a conical jet and the surrounding quiescent material, and are flowing along the surface of such a jet with velocities directed outward from the central YSO. Such an interpretation is fully confirmed by the proper motions of the $\mathrm{H}_{2} \mathrm{O}$ maser spots, shown in Fig. 2b: clearly, the spot velocities are diverging from the YSO. We stress again that in order to obtain the intrinsic proper motions of the spots (Cols. 8 and 9 of Table 1) the absolute proper motions (Cols. 6 and 7) have been corrected for the annual parallax and motion of the source relative to the Sun, assuming a distance of $1.7 \mathrm{kpc}$.

Combining the proper motions with the LSR velocity of each spot after subtraction of the systemic velocity of $-3.5 \mathrm{~km} \mathrm{~s}^{-1}$, one can obtain the corresponding speed of each spot. In Fig. 3 we plot this (filled circles) as a function of the distance $r$ (measured on the plane of the sky) from the peak of the $3.6 \mathrm{~cm}$ continuum emission, which is a good guess for the location of the YSO. Although with a large scatter, the speed tends to increase with $r$. A similar trend is seen for the 1.o.s. components of the spot velocities - which represent a lower limit to the full speed. These are shown in the same figure (empty circles) also for the spots that do not have measured proper motions. We conclude that the jet is accelerated within at least 700 au from the YSO.

For a more quantitative description of the maser distribution and velocities, in the next section we elaborate a simple model which represents an improvement on the model developed by MCR.

\section{A model for the $\mathrm{H}_{2} \mathrm{O}$ masers in IRAS $20126+4104$}

The scope of the jet model presented here is to prove that the scenario outlined in the previous section for the origin of the $\mathrm{H}_{2} \mathrm{O}$ maser emission in IRAS $20126+4104$ is correct. Unlike MCR's model, ours takes into account that the spot speed increases with distance from the YSO and fits also the velocity components in the plane of the sky. We assume that the maser spots lie on the surface of a cone with semi-opening angle $\theta$, inclination angle with respect to the 1.o.s. $\psi$, and vertex coincident with the YSO at $\alpha_{0}, \delta_{0}$. The spot velocities are directed radially outward from the vertex and are proportional to the distance, $R$, from it: $v(R)=\frac{\mathrm{d} v}{\mathrm{~d} R} R$. The coordinate system is centred on the vertex, with $z$ along the l.o.s. (the observer lies at $z=-\infty)$ and $x$ coincident with the projection of the jet axis on the plane of the sky. For further details and a sketch of the model we refer to Sect. 4.1 and Fig. 4 of MCR.

Under the previous assumptions one can express the components of the velocity along the axes as

$v_{\mathrm{x}}=\frac{\mathrm{d} v}{\mathrm{~d} R} x$

$v_{\mathrm{y}}=\frac{\mathrm{d} v}{\mathrm{~d} R} y$

$v_{\mathrm{z}}=\frac{\mathrm{d} v}{\mathrm{~d} R} z$

where $\frac{\mathrm{d} v}{\mathrm{~d} R}$ is constant and

$$
\begin{aligned}
z= & {\left[x \sin \psi \cos \psi\left(1+\cot ^{2} \theta\right)\right.} \\
& \left. \pm \tan \theta \sqrt{x^{2}+y^{2}\left(\cos ^{2} \psi-\cot ^{2} \theta \sin ^{2} \psi\right)}\right] \\
& \times\left(\cot ^{2} \theta \sin ^{2} \psi-\cos ^{2} \psi\right)^{-1}
\end{aligned}
$$

Note that Eq. (2) of MCR contains an unfortunate typing error $\left(\tan ^{2} \theta\right.$ must be replaced by $\left.\cot ^{2} \theta\right)$ which makes it different from our Eq. (5). The correct equation was used in the calculations of MCR.

The input parameters of the model are: the PA of the jet, $\theta, \psi, \frac{\mathrm{d} v}{\mathrm{~d} R}$, and the coordinates of the cone vertex, $\alpha_{0}$ and $\delta_{0}$. We searched for the values of these parameters minimising the expression

$\chi^{2}=\sum_{i=1}^{26}\left(v_{x}^{(i)}-V_{x}^{(i)}\right)^{2}+\left(v_{y}^{(i)}-V_{y}^{(i)}\right)^{2}+\left(v_{z}^{(i)}-V_{z}^{(i)}\right)^{2}$

where $i$ indicates the spot number in Table 1 and symbols $v$ and $V$ denote respectively the model and observed velocities; for spots without measured proper motions only the term involving the $z$ components is considered in the summation.

The best fit is obtained for PA $=123^{\circ}, \psi=96^{\circ}, \theta=$ $17^{\circ}, \alpha_{0}(\mathrm{~J} 2000)=20^{\mathrm{h}} 14^{\mathrm{m}} 26^{\mathrm{s}} .0410, \delta_{0}(\mathrm{~J} 2000)=41^{\circ} 13^{\prime} 32^{\prime \prime} \cdot 536$, and $\frac{\mathrm{d} v}{\mathrm{~d} R}=0.255 \mathrm{~km} \mathrm{~s}^{-1} \mathrm{mas}^{-1}=0.150 \mathrm{~km} \mathrm{~s}^{-1} \mathrm{au}^{-1}$. Figure 4 compares the measured values of the proper motions and LSR velocities (filled circles) with those obtained from the model fit (empty circles). Clearly the agreement is very good, proving that our model provides a satisfactory interpretation for the origin of the water masers in IRAS 20126+4104. 


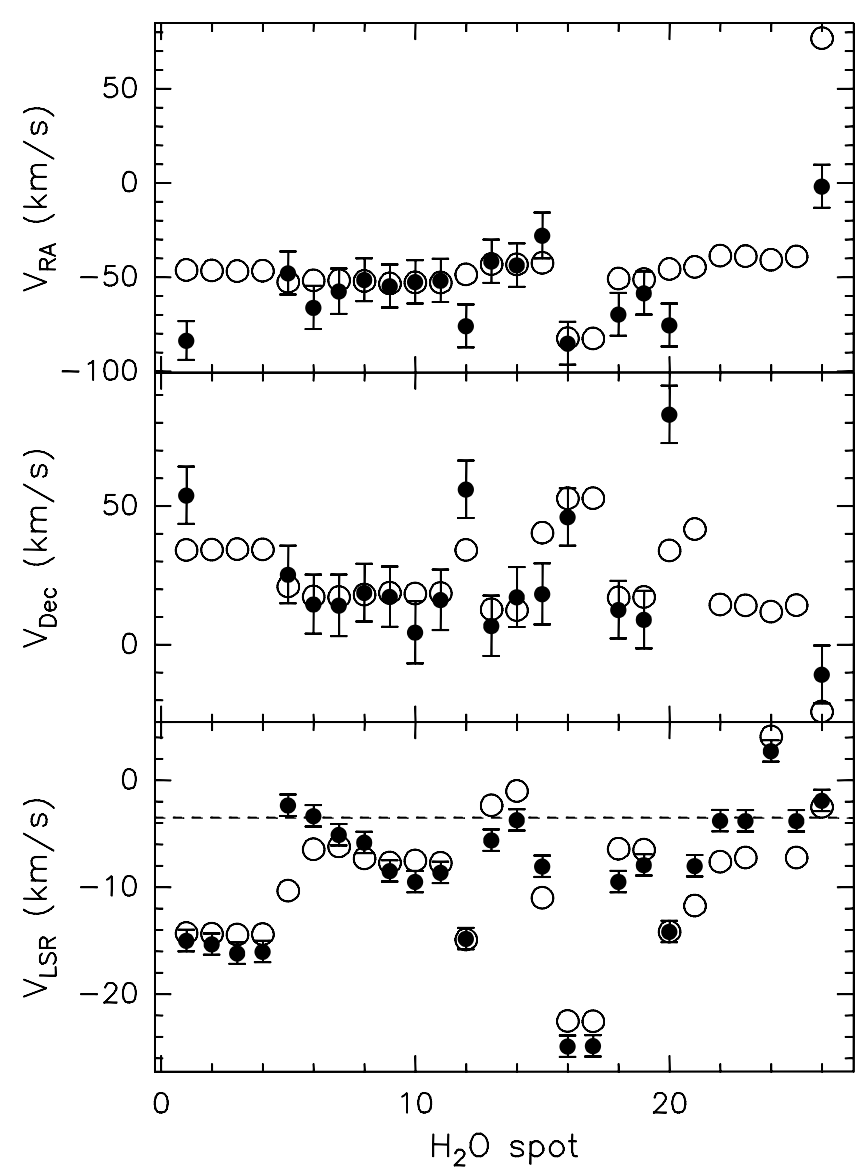

Fig. 4. Comparison between the observed velocities (filled circles) and those obtained from our model fit (empty circles). The top and middle panel show respectively the proper motions in the RA and Dec directions, while the bottom panel plots the LSR velocity. Note that data points missing in the top and middle panels correspond to spots without measured proper motions. The dashed line marks the systemic velocity $\left(-3.5 \mathrm{~km} \mathrm{~s}^{-1}\right)$ of IRAS $20126+4104$. The number on the $x$-axis indicates the maser spot according to the notation in Table 1 .

A comparison between our best fit parameters and those of MCR reveals significant differences, the most striking being the different expansion velocities: $23 \mathrm{~km} \mathrm{~s}^{-1}$ in MCR and $34-112 \mathrm{~km} \mathrm{~s}^{-1}$ in our case (see Fig. 3). This is due to the fact that the spots are moving almost in the plane of the sky, so that it is very difficult to correct for the inclination angle without proper motion information. As a matter of fact, $\psi$ is larger in our model than in MCR. Moreover, our value $\left(\psi=96^{\circ}\right)$ suggests that the north-western lobe of the jet is pointing away from us, whereas in $\operatorname{MCR}\left(\psi=59^{\circ}\right)$ the opposite occurs. Our result is consistent with recent findings: on the one hand, the jet is very close to the plane of the sky on scales as large as $10^{\prime \prime}$, or $0.08 \mathrm{pc}$ (Cesaroni et al. 1999); on the other hand, the jet axis is known to undergo precession, with the NW lobe receding from the observer at the present time (Cesaroni et al. 2005). It is hence not surprising that on scales as small as a few $100 \mathrm{au}$ the jet has already crossed the plane of the sky, as indicated by the value $\psi=96^{\circ}$.

As for the opening angle of the cone, MCR's estimate $\left(\theta=29^{\circ}\right)$ is twice as much as ours $\left(17^{\circ}\right)$. This is partly related to the fact that also the vertex of the cone has moved by $\sim 84$ mas to the SE with respect to MCR's estimate. As discussed in Sect. 3.3 the new position is closer to the disk plane, identified by the $\mathrm{OH}$ maser emission (see Fig. 2a).

In conclusion, we believe that the estimates of the $\mathrm{H}_{2} \mathrm{O}$ maser jet obtained in the present study represent an improvement on those of MCR and are hence to be preferred.

\section{Variability of the $\mathrm{H}_{2} \mathrm{O}$ masers}

It is interesting to complement the high resolution study of the spatial distribution of the $\mathrm{H}_{2} \mathrm{O}$ masers in IRAS 20126+4104 with an analysis of their time variability. Water masers are known to be highly variable and IRAS $20126+4104$ is no exception to this rule. Since 1987 , the Medicina 32-m antenna ${ }^{4}$ has been used as a single-dish telescope to monitor a number of galactic $\mathrm{H}_{2} \mathrm{O}$ masers with typical sampling intervals of 2-3 months. For details on the observations and data analysis we refer to Valdettaro et al. (2002). IRAS 20126+4104 belongs to this sample, so that we could retrieve the $\mathrm{H}_{2} \mathrm{O}$ spectra from the Medicina database and analyse them with the method developed by Valdettaro et al. (2002). This is shown in Fig. 5, which represents an image of the maser intensity as a function of time and LSR velocity. Also shown are the points corresponding to the peak velocities of the $\mathrm{H}_{2} \mathrm{O}$ lines detected at different epochs, including those obtained by us with global VLBI, by MCR with the VLBA, and by EFCE with MERLIN.

The most striking characteristic is the presence of three clear velocity trends, outlined by the dashed lines in Fig. 5: in all cases, the velocity decreases linearly with time, with slopes $\mathrm{d} V_{\mathrm{LSR}} / \mathrm{d} t \simeq-2.1,-2.7$, and $-2.1 \mathrm{~km} \mathrm{~s}^{-1} \mathrm{yr}^{-1}$ for features (1), (2), and (3) respectively. In the following we discuss these features in detail.

\subsection{Features (2) and (3)}

For features (2) and (3) one may easily derive the value of the deceleration corrected for the inclination angle. In fact, we have measured the proper motion of the corresponding spots: using the notation of Table 1 , these are spots 1,12 , and 20 for feature (2) and 16 for feature (3). The inclination angle is given by the ratio between the l.o.s. velocity and the projection of the velocity on the plane of the sky. Applying this correction, one obtains $\mathrm{d} V / \mathrm{d} t \simeq-18 \mathrm{~km} \mathrm{~s}^{-1} \mathrm{yr}^{-1}$ for (2) and $-9.7 \mathrm{~km} \mathrm{~s}^{-1} \mathrm{yr}^{-1}$ for (3).

In Fig. 6 the distribution of the spots corresponding to feature (2) is shown, including our measurements and those of MCR and EFCE. Notwithstanding the large positional errors on the VLBA and MERLIN measurements, the general trend follows the direction of the $\mathrm{H}_{2} \mathrm{O}$ maser jet depicted in our model. This is proved by the empty circles, which mark the positions of the spots with measured proper motions (filled circles with arrows) extrapolated at the two epochs of the VLBA and MERLIN observations. Such an extrapolation takes into account the acceleration in the plane of the sky estimated above.

4 The Medicina VLBI radiotelescope is operated by the INAF-Istituto di Radioastronomia. 


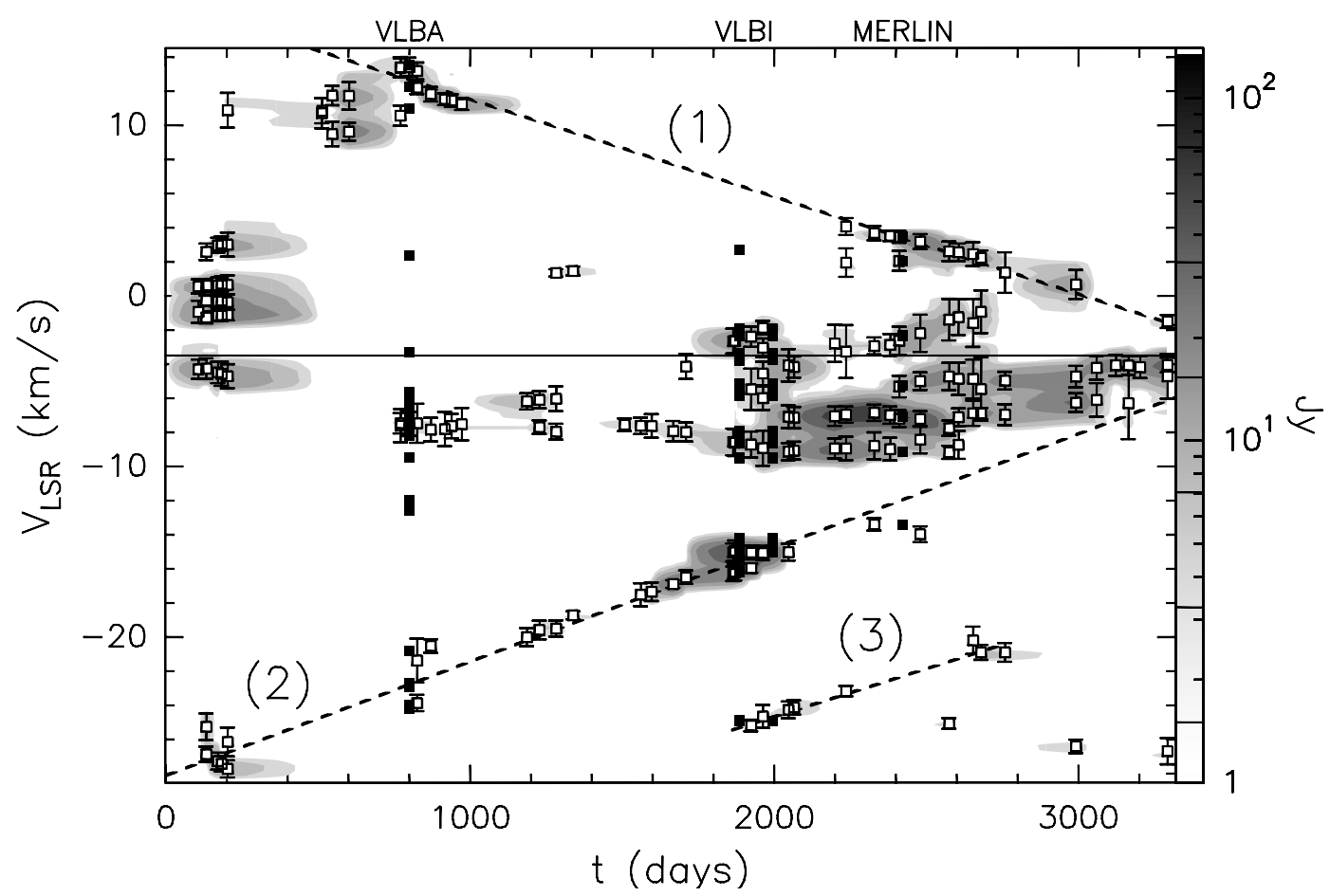

Fig. 5. Variability plot of the $\mathrm{H}_{2} \mathrm{O}$ maser emission in IRAS 20126+4104. The grey scale map represents the intensity measured with the Medicina antenna as a function of time and LSR velocity. The value $t=0$ corresponds to September 12, 1995. The white squares mark the positions of the the peak velocities of the lines detected in the Medicina spectra, while the error bars correspond to the line full width at half maximum $(F W H M)$. Both the peak velocity and line width have been obtained with a Gaussian fit. The black squares indicate the peak velocities of the spots observed in the VLBA, VLBI, and MERLIN observations of MCR, this study, and EFCE respectively. Only the first and third VLBI epochs are shown here to preserve readability of the figure. The horizontal solid line marks the systemic velocity $\left(-3.5 \mathrm{~km} \mathrm{~s}^{-1}\right)$ of IRAS $20126+4104$. The dashed lines outline the linear variation of velocity versus time observed in three spectral features, labeled with numbers (1) to (3).

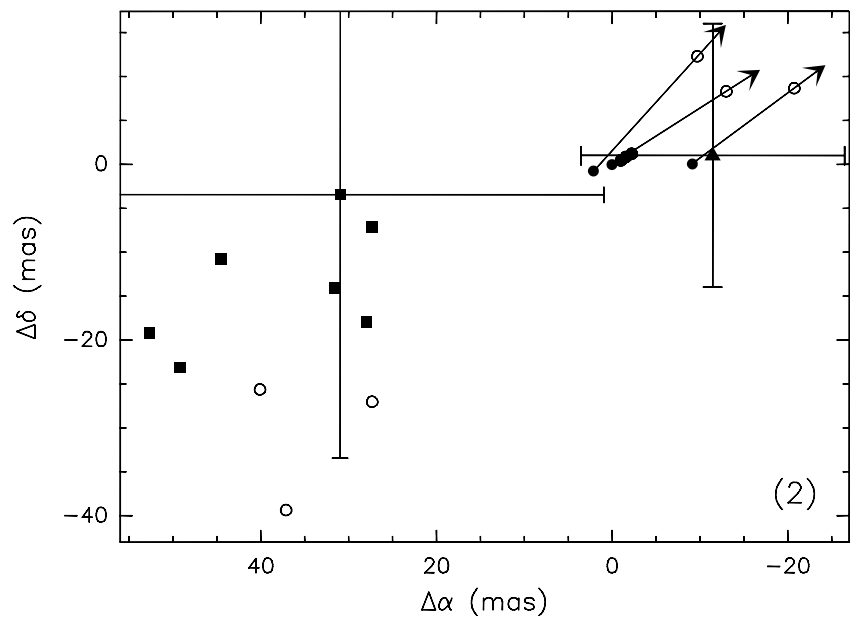

Fig. 6. Map of the $\mathrm{H}_{2} \mathrm{O}$ maser spots belonging to feature (2) in Fig. 5. The $(0,0)$ position corresponds to $\mathrm{RA}(\mathrm{J} 2000)=20^{\mathrm{h}} 14^{\mathrm{m}} 26^{\mathrm{s}} .0253$, $\operatorname{Dec}(\mathrm{J} 2000)=41^{\circ} 13^{\prime} 32^{\prime \prime}$.666. Black circles indicate our measurements, squares those by MCR, and triangles those by EFCE. The error bars indicate the $1 \sigma$ uncertainty in the astrometry of the MCR and EFCE measurements (the error on our data is less than the size of the symbols). Note that the error in the relative positions of the spots in each data set is of order 1 mas. The arrows denote the proper motions measured by us, while the empty circles mark the positions of the same spots extrapolated to the dates of MCR (November 21, 1997) and EFCE's (March 2002) observations, on the basis of the current spot position, proper motion, and acceleration (see text).
Clearly, the location of the empty circles is consistent within the errors with that of the VLBA (squares) and MERLIN (triangles) spots.

It is worth noting that the error bars in Fig. 6 represent the uncertainty on the absolute position at each epoch. The relative position of the spots observed at a given epoch is instead very accurate, of order $\sim 1$ mas. Therefore, it is clear that in the first epoch (squares) the dispersion between the spots was much greater than at the time of our VLBI observations. One may speculate that this an indication of the fact that the shocks associated with the masers are converging.

\subsection{Feature (1)}

Feature (1) has not been detected in our VLBI observations, so that no proper motion measurement is available. However, the VLBA and MERLIN measurements of MCR and EFCE can be used to obtain an estimate of the average proper motion. Notwithstanding the large uncertainty on the absolute positions of the spots, the time interval between the two measurements is large enough to guarantee an angular separation between the positions at the two epochs much larger than the errors. This can be seen in Fig. 7, where also a sketch of the conical model presented in Sect. 4 is shown for the sake of comparison. The mean velocity in the plane of the sky is obtained from the separation between the two positions ( $169 \pm 45$ mas or $287 \pm 77 \mathrm{au}$ ) 


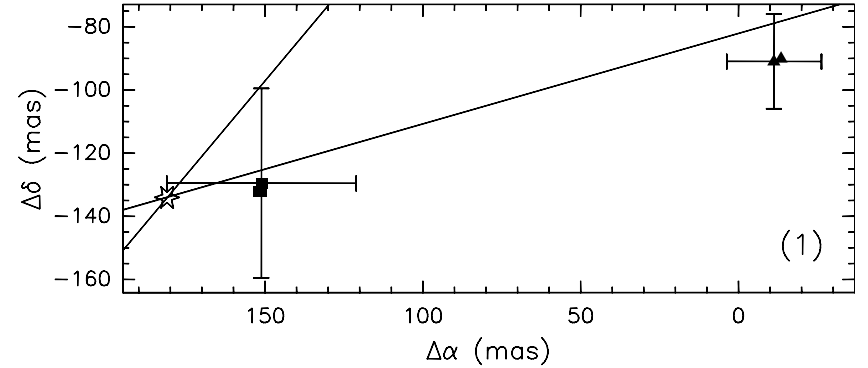

Fig. 7. Map of the $\mathrm{H}_{2} \mathrm{O}$ maser spots belonging to feature (1) in Fig. 5 . The $(0,0)$ position corresponds to $\mathrm{RA}(\mathrm{J} 2000)=20^{\mathrm{h}} 14^{\mathrm{m}} 26^{\mathrm{s}} .0253$, $\operatorname{Dec}(\mathrm{J} 2000)=41^{\circ} 13^{\prime} 32^{\prime \prime}$.666. Squares indicate measurements by MCR and triangles those by EFCE. The error bars indicate the $1 \sigma$ uncertainty on the astrometry. The error on the relative position of the spots in each data set is of order 1 mas. The starred polygon marks the position of the YSO according to our conical model fit, while the solid lines denote the projection of the cone on the plane of the sky.

divided by the corresponding time interval ( $\sim 1620$ days), and turns out to be $306 \pm 42 \mathrm{~km} \mathrm{~s}^{-1}$. We also stress that the direction of the proper motion is perfectly consistent with expansion along the cone as expected in our model: this has the twofold consequence of confirming our model and proving that all spectral features falling on the same dashed line in Fig. 5 do arise from a given spot (or group of spots) moving along the jet.

One may compute the mean velocity of feature (1) along the 1.o.s. from the arithmetic mean between the 1.o.s. velocities at the two epochs $\left(\sim 11.2 \mathrm{~km} \mathrm{~s}^{-1}\right)$. Finally, from the ratio between this and the proper motion, it is possible to obtain the inclination of the velocity vector with respect to the 1.o.s. and thus correct the 1.o.s. values of the mean speed $\left(11.4 \mathrm{~km} \mathrm{~s}^{-1}\right)$ and acceleration $\left(-2.1 \mathrm{~km} \mathrm{~s}^{-1} \mathrm{yr}^{-1}\right)$ : the corrected values are $306 \mathrm{~km} \mathrm{~s}^{-1}$ and $-56 \mathrm{~km} \mathrm{~s}^{-1} \mathrm{yr}^{-1}$.

\subsection{Origin of the deceleration}

From the previous findings, we conclude that $\mathrm{H}_{2} \mathrm{O}$ masers in IRAS 20126+4104 are undergoing a strong deceleration ranging from -50 to $-10 \mathrm{~km} \mathrm{~s}^{-1} \mathrm{yr}^{-1}$. Although evidence of deceleration from single-dish monitoring of $\mathrm{H}_{2} \mathrm{O}$ masers has already been reported by other authors (see Brand et al. 2003), to our knowledge this is the first time that such a finding is confirmed by high-angular resolution observations.

This existence of deceleration may look contradictory with the positive velocity trend shown in Fig 3 . However, $\mathrm{d} V / \mathrm{d} R>0$ does not necessarily imply $\mathrm{d} V / \mathrm{d} t>0$, because the $V(R)$ provided by our VLBI measurements corresponds to an instantaneous picture of the spots velocity field, whereas $V(t)$ obtained from the variability analysis describes the evolution of each single spot with time.

In order to reconcile $\mathrm{d} V / \mathrm{d} R>0$ with $\mathrm{d} V / \mathrm{d} t<0$, we propose the following scenario. Water masers are very likely generated in the post-shock region of J-type shocks (Elitzur et al. 1989). In our model, such shocks occur along the conical surface representing the interaction between the jet and the surrounding cloud. As predicted by theoretical models (e.g. Ferreira \& Casse 2004; Shu et al. 2000), the jet should be

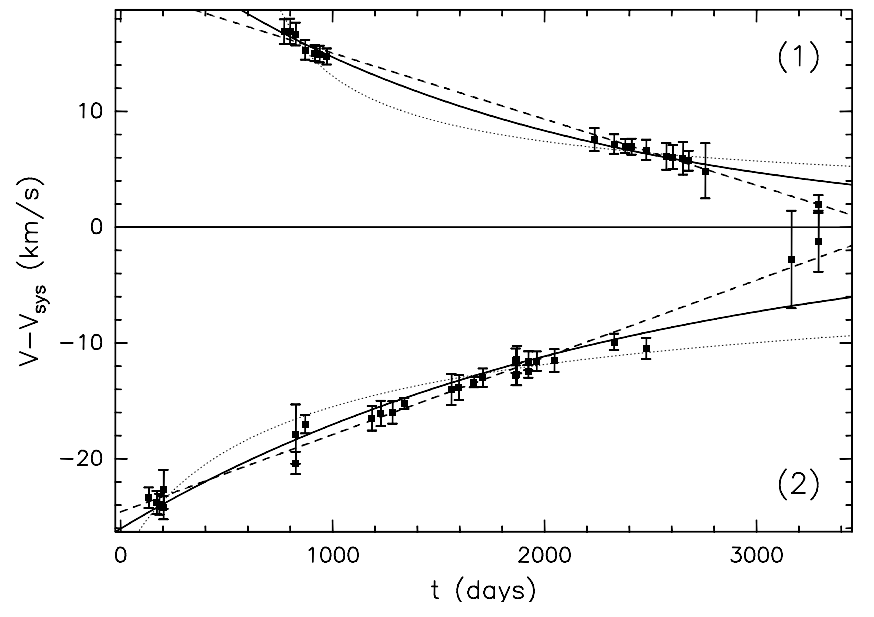

Fig. 8. Peak velocity of feature (1) and (2) (see Fig. 5) as a function of time. Error bars denote line FWHMs. The dashed lines are the same as in Fig. 5 and represent linear fits to the data, while dotted and solid lines are fits of the type $V \propto\left(t-t_{0}\right)^{-1 / 2}$ and $V \propto \exp \left[-\left(t-t_{0}\right) / \tau\right]$, respectively.

accelerated close to the YSO. Consequently, the further away (from the YSO) the jet impinges against the surrounding material, the larger will be the velocity of the corresponding shock. At this time a maser spot "is switched on", with the same velocity as the shock. This explains why $\mathrm{d} V / \mathrm{d} R>0$. After that, the shock is slowed down as it proceeds through the dense material of the cloud, thus causing the observed deceleration, $\mathrm{d} V / \mathrm{d} t<0$, of the spot velocity.

It remains to be verified that the scenario depicted above can fit the observed trend of $V(t)$ quantitatively. If the deceleration is due to braking of a shock, this should imply a power-law for $V(t)$. In fact, for shocks conserving energy one has $M V^{2}=$ const. $\Rightarrow V t V^{2}=$ const. $\Rightarrow V \propto t^{-1 / 3}$, while shocks conserving momentum imply $M V=$ const. and hence $V \propto t^{-1 / 2}$. Another possibility is that of an exponential decay of the shock velocity: naïvely, this may be described with a sort of "viscosity" in the medium ahead of the shock. With this in mind, we have fitted the velocity trends of features (1) and (2) with $V \propto\left(t-t_{0}\right)^{-1 / 2}$ and $V \propto \exp \left[-\left(t-t_{0}\right) / \tau\right]$ : these are represented respectively by the dotted and solid curves in Fig. 8, where we plot the l.o.s. velocities of features (1) and (2) as a function of time. Also shown are linear fits to the data (dashed lines). It is worth noting that in all cases the lifetime of the maser spots ranges between 5 and $12 \mathrm{yr}$, a timescale comparable to our monitoring period. Clearly, it is difficult to rule out any of the models, although the exponential fit seems more satisfactory. A more sensitive and frequent monitoring of the $\mathrm{H}_{2} \mathrm{O}$ maser emission in IRAS $20126+4104$ is necessary to discriminate between models.

\section{Summary and conclusions}

We have performed three-epoch VLBI observations of the $\mathrm{H}_{2} \mathrm{O}$ maser emission from the massive protostar IRAS 20126+4104. Phase referencing allows us to measure the absolute proper motions of the maser spots and hence obtain a 3-D picture of the velocity field. These high 
angular resolution results have been complemented by an analysis of the variability of the $\mathrm{H}_{2} \mathrm{O}$ maser emission through single-dish observations spread over 15 years. The main results obtained from this study and comparison with previous data by MCR and EFCE are the following:

- A total of 26 spots have been detected in our VLBI observations, 17 of which at all three epochs thus allowing measurement of their proper motions.

- An attempt to use proper motions to obtain an estimate of the distance to the source may only loosely constrain it between 1.2 and $2.7 \mathrm{kpc}$.

- After correcting the measured proper motions for annual parallax and apparent motion of the source with respect to the Sun (assuming a distance of $1.7 \mathrm{kpc}$ ), we find that the maser spots are expanding from a common origin, consistent with the expected position of the protostar. The speed is increasing from 34 to $112 \mathrm{~km} \mathrm{~s}^{-1}$ for increasing distance from the protostar.

- The 1.o.s. velocities and proper motions of the spots can be fitted with an improved version of the conical jet model proposed by MCR in a previous VLBA study of the same maser source. The best fit is obtained for a jet semi-opening angle of $17^{\circ}$, an inclination of $96^{\circ}$ with respect to the 1.o.s. (the jet axis to the NE is pointing away from the observer), an expansion velocity gradient of $255 \mathrm{~km} \mathrm{~s}^{-1} \mathrm{yr}^{-1}$, and a cone vertex located at $\mathrm{RA}(\mathrm{J} 2000)=20^{\mathrm{h}} 14^{\mathrm{m}} 26^{\mathrm{s}} .0410$, $\operatorname{Dec}(\mathrm{J} 2000)=41^{\circ} 13^{\prime} 32^{\prime \prime} .536$. This is consistent with the expected position of the protostar.

- The variability study demonstrates that some of the maser features undergo decelerations from $\sim-50$ to $-10 \mathrm{~km} \mathrm{~s}^{-1} \mathrm{yr}^{-1}$, with lifetimes of the order 5-12 $\mathrm{yr}$. We speculate that this may be due to braking of the shocks where the maser emission is originating, at the interface between an accelerated jet and the surrounding molecular environment.

We conclude that our study has shed light on the 3-D structure and kinematics of the jet in IRAS 20126+4104 and outlined some intriguing characteristics of the time variability of the maser - and hence of the jet. A full understanding of this latter aspect will require more sensitive observations with better angular and, possibly, temporal resolution.
Acknowledgements. It is a pleasure to thank Prof. Tetsuo Sasao for allowing us to use his program for the computation of apparent proper motions due to annual parallax, solar motion with respect to the LSR, and galactic rotation. We are also grateful to Gary Fuller for making available the results of his MERLIN observations, prior to publication. Many thanks are due to Francesco Palla and Jan Brand for a critical reading of the manuscript and Daniele Galli and Rino Bandiera for stimulating discussions.

\section{References}

Brand, J., \& Blitz, L. 1993, A\&A, 275, 67

Brand, J., Cesaroni, R., Comoretto, G., et al. 1993, A\&A, 407, 573

Cesaroni, R., Felli, M., Testi, L., Walmsley, C. M., \& Olmi, L. 1997, A\&A, 325, 725

Cesaroni, R., Felli, M., Jenness, T., et al. 1999, A\&A, 345, 949

Cesaroni, R., Neri, R., Olmi, L., et al. 2005, A\&A, 434, 1039

Edris, K. A., Fuller, G. A., Cohen, R. J., \& Etoka, S. 2005, A\&A, 434, 213 (EFCE)

Elitzur, M., Hollenbach, D. J., \& McKee, C. F. 1989, ApJ, 346, 983

Elitzur, M. 1992, Astronomical Masers (Dordrecht: Kluwer Academic Publisher)

Felli, M., Palagi, F., \& Tofani, G. 1992, A\&A, 255, 293

Ferreira, J., \& Casse, F. 2004, Ap\&SS, 292, 479

Goddi, C., Moscadelii, L., Alef, W., \& Brand, J. 2004, A\&A, 420, 929

Hofner, P., Cesaroni, R., Rodríguez, L. F., \& Martí, J. 1999, A\&A, 345, L43

Moscadelli, L., Cesaroni, R., \& Rioja, M. J. 2000, A\&A, 360, 663 (MCR)

Reid, M. J., Schneps, M. H., Moran, J. M., et al. 1988, ApJ, 330, 809

Shepherd, D. S., Yu, K. C., Bally, J., \& Testi, L. 2000, ApJ, 535, 833

Shu, F. H., Najita, J. R., Shang, H., \& Li, Z.-Y. 2000, Protostars and Planets IV, ed. V. Mannings, A. Boss, \& S. Russel (Tucson: Univ. of Arizona Press)

Torrelles, J. M., Gómez, J. F., Rodríguez, L. F., et al. 1996, ApJ, 457, L107

Torrelles, J. M., Gómez, J. F., Rodríguez, L. F., et al. 1998, ApJ, 505, 756

Torrelles, J. M., Patel, N. A., Gómez, J. F., et al. 2001, Nature, 411, 277

Valdettaro, R., Palla, F., Brand, J., et al. 2002, A\&A, 383, 244

Vlemmings, W. H. T., van Langevelde, H. J., Diamond, P. J., Habing, H. J., \& Schilizzi, R. T. 2003, A\&A 407, 213

Zhang, Q., Hunter, T. R., \& Sridharan, T. K. 1998, ApJ, 505, L151 